\title{
An Overview on a Technique to Measure and Control of the Electromagnetic Radiation Inside a Microwave Oven
}

Farah A.Thamer ${ }^{1}$, Haitham B. Al-Wakeel ${ }^{2}$, Taghreed Mohammadridha ${ }^{1}$, Zeyid T. Ibraheem ${ }^{2}$

${ }^{1}$ Control and Systems Eng. Dept. University of Technology, Baghdad, Iraq

${ }^{2}$ Directorate of Industrial Research and Development, Ministry of Science and Technology, Baghdad, Iraq Email:farah96control@gmail.com, haitham_alwakel@yahoo.com,60034@uotechnology.edu.iq, zeyidtariq@gmail.com

\begin{abstract}
The Microwave oven is a system used to convert the electromagnetic energy to thermal energy when the microwave cavity is loaded with a dielectric material. The ordinary microwave ovens are not supported with complex features for detecting parameters such as temperature, weight, and loaded material availability. Due to the lack of material availability, several laboratory and industrial applications require these features to switch off the oven. The reflections of electromagnetic radiation inside an empty microwave oven lead to oven damage. An overview of the microwave oven characteristics and emergence of electromagnetic radiation inside a microwave oven is presented in this study. The parameters measured inside the microwave oven, methods for power attenuation in a microwave oven, microwave power detector, and microwave oven leakage are discussed as well. Moreover in the methodology of this work, proposed a new technique based on the measurement of leaked microwave power to control the microwave oven. The preliminary results showed that the leakage measurement of electromagnetic power changes with the state/phase of the material inside the microwave oven, which ensured the possibility of the proposed promising technique. This work will be continued to connect the microwave oven with a spectrum analyzer and computer via hardware and software interfaces depending on the methodology of this article. A computer code will be developed to read the measured power and automatically switch off the microwave oven depending on materials state.
\end{abstract}

Index Terms-Microwave, power, measurement, control.

LIST OF NOMENCLATURE

\begin{tabular}{|l|l|}
\hline$P_{\text {abs }}$ & Absorbed power \\
\hline$P_{\text {in }}$ & Power in \\
\hline$P_{\text {out }}$ & Power uot \\
\hline Tan $\boldsymbol{\delta}$ & Dielectric loss tangent \\
\hline $\boldsymbol{J H E A T}$ & Dissipated or generated heat \\
\hline $\boldsymbol{E}$ & Electric field intensity vector \\
\hline $\boldsymbol{E}_{\mathbf{0}}$ & Amplitude of electric field intensity \\
\hline $\boldsymbol{K}$ & power attenuation of microwave cavity \\
\hline $\boldsymbol{S}$ & antenna sensitivity \\
\hline $\boldsymbol{\mu}$ & Magnetic permeability \\
\hline
\end{tabular}


DOI: https://doi.org/10.33103/uot.ijccce.21.1.4

\begin{tabular}{|l|l|}
\hline$\varepsilon$ & Complex permittivity or electric permittivity \\
\hline$\varepsilon_{0}$ & Permittivity of vacuum or air $(\mathbf{8 . 8 5 4 \times 1 0 - 1 2 ~} \mathbf{~} / \mathbf{m})$ \\
\hline$\sigma$ & Electric conductivity \\
\hline $\boldsymbol{w}$ & Angular frequency \\
\hline $\boldsymbol{f}$ & Operating frequency of a wave \\
\hline$f_{\boldsymbol{c}}$ & Cutoff frequency \\
\hline$\varepsilon_{\mathrm{r}}{ }^{\prime}$ & Relative dielectric constant \\
\hline$\varepsilon_{\mathrm{r}}{ }^{\prime \prime}$ & Relative dielectric loss factor \\
\hline$Z$ & Intrinsic impedance for medium \\
\hline$Z_{x y}$ & Transverse waves impedance \\
\hline a,b & Width and height of wave incident plane \\
\hline$H_{0}$ & magnetic field \\
\hline
\end{tabular}

\section{INTRODUCTION}

Electromagnetic radiation is a phenomenon that takes the form of a wave that can propagate in the vacuum and materials [1]. The Electromagnetic Wave is a coupling between the unstable vectors of the electrical field and the magnetic field. The two fields spread out from each other, aligned with time and space at the right angles as a wave moving in the same direction as shown in Fig. 1. Thus the wave has one part of the electric field and one part of the magnetic field, which are transversal to the propagation direction. The electromagnetic waves include radio waves, microwave, infrared, light, ultraviolet, $\mathrm{x}$ ray, and Gama ray. Microwaves are part of the electromagnetic spectrum having a range of frequencies $300 \mathrm{MHz}$ to $300 \mathrm{GHz}$ [1]. The microwaves are capable of penetrating through dielectric materials. The electromagnetic power of microwaves inside a closed microwave cavity is absorbed by dielectric materials and lost as heat to achieving rapid volumetric heating, which is opposed to conventional heating that heats the surfaces. The domestic microwave ovens work at a $2.45 \mathrm{GHz}$ based frequency [2]. Several types of research used successfully the microwave heating and microwave oven for domestic and industrial applications. However, there are problems related to measurements of materials loaded inside the microwave oven. Such as, the problem of microwave heating for soot filter regeneration [3], which is how to differentiate if the filter is empty or full of soot because the reflection of the electromagnetic waves inside an empty closed microwave cavity is dangerous for the magnetron. The problem of fruits, vegetables [4], and seeds [5] drying by microwave heating, which is how to assess microwave power rates in microwave dryers with and without temperature regulation. Where the microwave dries the materials from inside, but the temperature sensor measures the outside surface. The problem of domestic microwave oven [6], is not having a measurement for the state of the loaded material. These problems can be solved by providing a technique for automatic measurement and control on the electromagnetic radiation inside a microwave oven. This article presents an understanding of the details of the oven working by the microwave radiation and behaviors of electromagnetic radiation inside a microwave oven. Besides, shows a new concept for microwave power measurement to automatically switch off the microwave oven, response for material state. This article is organized as in following. Section II describes the advantages of heating by microwaves. Section III presents the microwave oven features. Section IV demonstrates electromagnetic radiation inside a microwave oven. Section V shows the parameters measure inside the microwave oven. Methods used for attenuation of 
electromagnetic power inside a microwave oven are discussed in Section VI. Section VII presents the microwave detector. In Section VIII microwave leakage is presented. Section IX presents the automatic control of the power of the microwave oven. Section X presents the methodology proposed for automatic control on the microwave oven. In Section XI experimental work is presented. Section XII shows the preliminary results of this work.

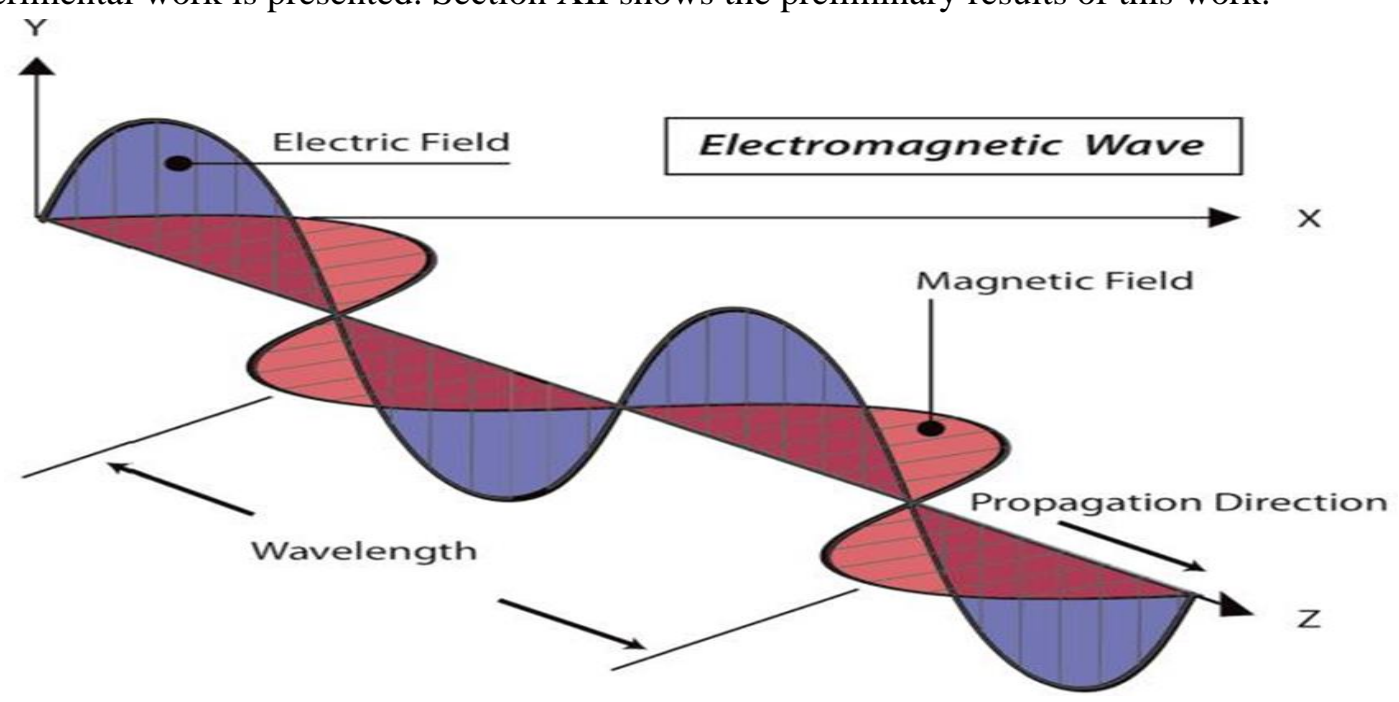

Fig. 1. A PROPAGATING ELECTROMAGNETIC WAVE.

\section{ADVANTAGES OF MICROWAVE HEATING}

The benefits of using microwave heating include volumetric heating, which ensures that materials can directly and effectively consume microwave energy and rapidly transform it to heat [7]; accelerated heating decreases thermal gradients and stresses [8]; efficient and fast transfer of energy decreeing the time of processing [9]; control in the source of power; selective heating through differential penetration of materials; self-limiting reaction [10]; inverted temperature gradient (heating from inside to outside); quick-drying moisture [11]. The downside is that the non-homogeneous distribution and transfer of energy in the microwave cavity generates non-uniform gradients of temperature rise [12].

Microwave heating has been used by many researchers to help the catalyst lower the chemical reaction temperature or raise the soot temperature to regenerate the soot trap[9]. Although, some used the catalyst to support microwave creating non-thermal plasma, eliminating contamination sources due to the lack of electrodes, and ensuring easy and fast control on the processes [13].

\section{MICROWAVE OVEN CHARACTERISTICS}

The microwave ovens are metal boxes equipped with glass doors supported with metal nets that attenuate the microwave radiation leakage [14]. The oven derives its name from the radiation that cooks food and is called the microwave radiation. Microwave is part of the electromagnetic wave group. One of the key features of the microwave oven is that excites molecules, makes them vibrate and heat up from inside to outside. That is why food is cooked very quickly [15]. The heart of this device is the magnetron. The waveguide guides electromagnetic waves to the cavity of the microwave oven [16]. An oven includes a cooking chamber configured to receive a food product, a user interface configured to display information associated with processes employed for cooking [17]. Microwave absorption efficiency differs greatly depending on the object being heated [18]. A control system for a microwave controller includes a programmable logic control circuit, which enables the user to program information of cooking power and time. The user program information 
into the control by means of a touch plate, which is sectioned off into a plurality of areas, each area representing certain information to be entered into the control. The information, which is entered by the user, is displayed in the digital oven to provide visual feedback to the user [19].

\section{ELECTROMAGNETIC RADIATION INSIDE A MICROWAVE OVEN}

The electromagnetic radiation inside the closed cavity of microwave oven is absorbed by dielectric materials and dispelled as thermal energy. The absorbed electromagnetic power that is dissipated as heat, is formulated in relation to the electrical field, electromagnetic frequency, and dielectric properties as shown in (1) [20, 21].

$$
P_{a b s}=\left(\frac{1}{2}\right) 2 \pi f \varepsilon_{\mathrm{o}}\left(\varepsilon_{\mathrm{r}}{ }^{\prime}+\frac{\sigma}{\omega \varepsilon_{\mathrm{o}}}\right)|E|^{2} d V
$$

where, $E$ is electric field vector, $\mu$ is magnetic permeability, $\varepsilon$ is electric permittivity, $\varepsilon_{0}$ is a permittivity of vacuum or air $(8.854 \times 10-12 \mathrm{~F} / \mathrm{m}), \sigma$ is electric conductivity, $w$ is angular electromagnetic frequency and, $f$ is operating frequency.

The measurement of the dielectric material ability to absorb an electromagnetic energy and convert it to a thermal energy depends on dielectric loss tangent, $\tan \delta$. Tan $\delta$ is determined from the relative dielectric constant, $\varepsilon_{\mathrm{r}}{ }^{\prime}$, and relative dielectric loss factor, $\varepsilon_{\mathrm{r}}{ }^{\prime \prime}$, according to (2).

$$
\tan \delta=\left(\varepsilon r^{\prime \prime} / \varepsilon r^{\prime}\right)+\sigma /\left(\omega \varepsilon_{\mathrm{o}} \varepsilon r^{\prime}\right)
$$

The dielectric loss factor shows a material's capacity for dissipating the accumulated energy to heat. Electrical conductivity is zero for a dielectric material, but it is in the range of $10^{7}$ for metals [20].

\section{PARAMETERS MEASURED INSIDE THE MICROWAVE OVEN}

The following subsections reviewed studies focused on parameters measured inside the microwave oven:

\section{a. Temperature}

Microwave drying technology is used in large industrial applications, given the various advantages of traditional drying systems[22]. During microwave drying, especially in products with high moisture content such as fruits and vegetables, the temperature increases dramatically as they respond greatly to microwave absorption. The more energy that substance consumes the higher of temperature. With unregulated temperature, the material properties can change, resulting in thermal burning and non-uniform distribution of temperature in the materials being treated. To ensure effective monitoring of the magnetron engine without material degradation, an automatic phase control on the basis of temperature measurement was proposed in [4]. The remote temperature measurement of edible materials that were heated inside microwave ovens was revealed in [23].The proposal contains a probe attached to a temperature-sensitive circuit to produce a signal linked with an electromagnetic wave transmitting circuit placed in the microwave oven for telemetric transmission of the signal to a remote receiver and coupled to a control circuit.

\section{b. Weight}

A design for controlling the microwave oven for daily use was proposed by (Alrazaaq, 2016). The goal of this proposal was to improve the operation of the microwave oven. Three features were introduced to complement the current design. The first feature was to add a load cell to calculate the weight of the processed food under a turntable specified for microwave oven. The second feature was to create a fuzzy controller which 
can determine automatically the appropriate level of power and heating time according to the calculation of weight. The third feature was to mount a steam sensor inside the microwave oven's cavity to detect the occurred steam to help the controller to calculate the remaining time to complete. The findings showed that the successful operation of the design can be done by putting the right parameters for food processing without relying on repeated estimates [24].

\section{c. Electromagnetic power}

The electromagnetic power is the measure of signal intensity within the spectrum of radiofrequency and microwave frequency. This is stated in Watts or $\mathrm{dBm}$. The (0) $\mathrm{dBm}$ denotes to (1) $\mathrm{mW}$ according to the logarithmic value given in (3) [25].

$$
P_{d B m}=\log _{10}(P / 1 m W)
$$

The electromagnetic power of frequencies spectrum $2.4-2.5 \mathrm{GHz}$ inside a microwave cavity without magnetron was measured using Network Analyzer [21]. As well, the microwave spectrum inside a microwave oven unattached with a magnetron, loaded with soot and thermocouple was measured in [3] using a spectrum analyzer and portable network analyzer. The results showed two resonance frequencies of 2.45 and $2.425 \mathrm{GHz}$ and no effective loss in the incident microwave power due to the reflection in the soot and thermocouple wire. However, Electromagnetic power that is output from magnetron of microwave oven is about 700 to $900 \mathrm{~W}$. But the limitations of the spectrum analyzer are to analyze the electromagnetic frequencies carried with the power of less than $1 \mathrm{~W}$. Therefore, power attenuation should be equipped to measure the electromagnetic power inside the microwave cavity. Methods used for electromagnetic power attenuation in a microwave oven are discussed in the next section.

\section{METHODS USED FOR ATTENUATION OF ELECTROMAGNETIC POWER INSIDE A MICROWAVE OVEN}

There are several methods used to attenuate the electromagnetic power inside the microwave cavity:

\section{d. Microwave attenuator}

A system of the silicon-based photoconductive switch and microwave attenuator was optimized for low insertion loss and elevated attenuation for light emitted from the infrared LED. The system is integrated into the coplanar waveguide and a planar antenna for controlling the photonic array. The improved performance of the system was up to $-20 \mathrm{~dB}$ attenuation, with only $0.6 \mathrm{~dB}$ insertion loss at $2.0 \mathrm{GHz}$ frequency. For a range of frequencies, the insertion phase was changed slightly upon attenuation by less than $1 \% \mathrm{~dB}$ [26]. The reconfigurable attenuators have been commonly used in the measuring instruments for microwaves. The production of miniaturized attenuators with high precision and efficiency becomes required for recent applications. The manufacturing of reconfigurable attenuators that are integrated with RF MEMS switches and resistive attenuation modules with dimensions of $(2.45,4.34,0.5) \mathrm{mm} 3$, which is $1 / 1000$ th of the size of a traditional phase attenuator is presented in [27].

\section{e. Attenuation by absorption load}

A procedure for measuring the energy absorption by a material placed in a microwave oven was proposed in [28] operating at high power levels was based on the determination of the relation between the power of the incident signal and the power of the reflected signal in a waveguide structure of high-power. 
Two microwave systems are known as the insertion transmission method and the free space transmission method to measure the power absorption was implemented in [29]. The effect of tissue type, moisture content, and separation between the transmitting and receiving horn Antennas were taken into account. Practical findings were conveyed through the application of Microsoft Excel software using the program kit [29]. In [30] two main models of electromagnetic attenuation were developed to measure the amount of attenuation for an electromagnetic signal. The names of these models were modified-Friis model, and the complex refractive index model (CRIM-Fresnel). Laboratory results revealed that the CRIM-Fresnel model offers a better attenuation in specimens of low permittivity whereas the modified-Friis model was more reliable in specimens of high water content with high permittivity. These methods were developed to measure electromagnetic signals attenuation in the laboratory [30]. However, properties, position, size, and shape of the absorbed load (dielectric sample) affect the distribution of the electric field and dissipated heat inside a microwave cavity [31].

\section{MICROWAVE DETECTOR}

A system of microwave detector probe was used to monitor the electromagnetic fields in the microwave ovens. Although the electromagnetic field is not visible inside the microwave oven, the magnetron is currently a tiny, strong, and solid-state furnace that can track the power that is transferred to foods. The microwave detector system, which was antenna and rectifier was inserted inside a microwave cavity loaded with dielectric materials, converted the unabsorbed power of $2.45 \mathrm{GHz}$ frequency to a DC voltage [32].

\section{MICROWAVE LEAKAGE}

This section evident that there is a microwave leakage from a microwave oven contrary to what is commonly known. A comparison of the values of electromagnetic field emissions experimentally from three types of microwave ovens was studied in [33]. Differences in electromagnetic radiation inside and outside the run-time ovens were established. The data that were collected at various distances from the microwave ovens and in different positions indicated to there is a radiation leakage during the service than values that were given in the regulations for all types of ovens being studied. Within a complex indoor scenario, electromagnetic field leakage rate as nonionizing radiations from microwave ovens was investigated [34]. It was possible to obtain estimates of the value of electrical fields for the indoor scenario by using a hybrid simulation depending on coupling full wave simulation with the in-house developed deterministic three dimensional ray launching an application. Microwave ovens could be simulated as time and frequency dependent radiating sources. The electromagnetic leakage was propagated, essentially from doors of microwave ovens. This method assisted to evaluate the effect of such devices on the expected level of exposure, permitting for the application of adequate minimization strategies such as optimal location to be applied. A micro-strip band-stop filter (BSF) was simulated, implemented, engineered, and tested as a trial to reduce the microwave leakage from a $2.45 \mathrm{GHz}$ domestic microwave oven [35]. The results showed that the simulated filter offered high selectivity for a stop-band response. The prototype of the simulated filter was produced. The limitation was the size of the microwave oven openings, which was counted in the simulation. Therefore, the methodology of automatic control that is proposed in Section $\mathrm{X}$ of this study is based on measuring the power of microwave leaked from the microwave oven. 


\section{AUTOMATIC CONTROL ON POWER OF MICROWAVE OVEN}

The automatic control of electromagnetic radiation inside a microwave oven, which is part of the topic of this study, can be applied by controlling the power supplied to operate the microwave oven. Hence, one of the applications is using the Arduino microcontroller to control the power of electric heating devices based on measurement parameters that are mentioned in Section V. The widely used control strategy in the industry is the proportional integral derivative (PID) controller [36]. A system of microwave heating equipped with a (PID) control was developed as described in [37] for the in-package pasteurization of readyto-eat meats called Frankfurts. Two types of heating, microwave, and water immersion heating were subjected to Frankfurters which were inoculated and vacuum-sealed in containers of plastic. The surface temperature of the product was sustained at 65, 75, and $85^{\circ} \mathrm{C}$ for different time periods. The total heating time was ranging from 2 to $19 \mathrm{~min}$. The surface temperature of the frankfurters packages during microwave heating increased linearly with the heating time until it reached a set point. This research proved the objective of the PID-controlled microwave heating for pasteurizing of frankfurters in-package. A fast feedback control strategy for a microwave power level control was proposed in [38]. The sample temperature control used a bang-bang control and PID strategy. Further, several experiments were performed, and experimental results showed that the output microwave power was accurate to the setpoint, and the PID algorithm resulted in the best control of the sample temperature compared with the bang-bang strategy. In [39] a control system was developed by connecting the phase-controlled electrical power regulator in series to an existing domestic microwave oven's original cycle-controlled power regulator. The microwave oven was further adjusted so that it can handle microwave combined with traditional drying. It was noticed that for quasi-continuous (fast-switching) power regulation, the phase-controlled power regulator could be utilized efficiently with optimization for power performance. A domestic microwave oven of 700 Watt was modified in [6], utilizing a TRIAC to control the magnetron input power. The resultant of microwave power output from the magnetron was measured to evaluate the initial system. More modification was done with a view of improving the figures of merit. A phasemicrocontroller device was built as a power control of an efficient, continuous, and smooth variation of power for the magnetron, depending on a feedback signal of the temperature of treated goods [40].

\section{METHODOLOGY OF AUTOMATIC CONTROL ON MICROWAVE OVEN}

The system structure of automatic control on microwave oven that is shown in the block diagram of Fig. 2 Includes:

a) Input; which is a reference or set point of electromagnetic power measured in $\mathrm{dbm}$ at empty microwave oven.

b) Process or control; which compares the power values of input with feedback in relation to time till arrive zero error.

c) Output; which is an actuated signal to switch off the microwave oven automatically at zero error.

d) Feedback; which is the timely measuring of electromagnetic power in $\mathrm{dbm}$ of loaded operating microwave oven.

The methodology for automatic control on microwave oven of this research is based on a new proposed technique used as a system feedback, which is the measurement of electromagnetic power leakage of microwave oven. In this work, Arduino Uno SDM of a low power consumption with the main controller Atmega 328 will be used to switch off 
DOI: https://doi.org/10.33103/uot.ijccee.21.1.4

the oven depending on the comparisons of leakage power values in relation with time that are indicated by the feedback signal till arriving zero error.

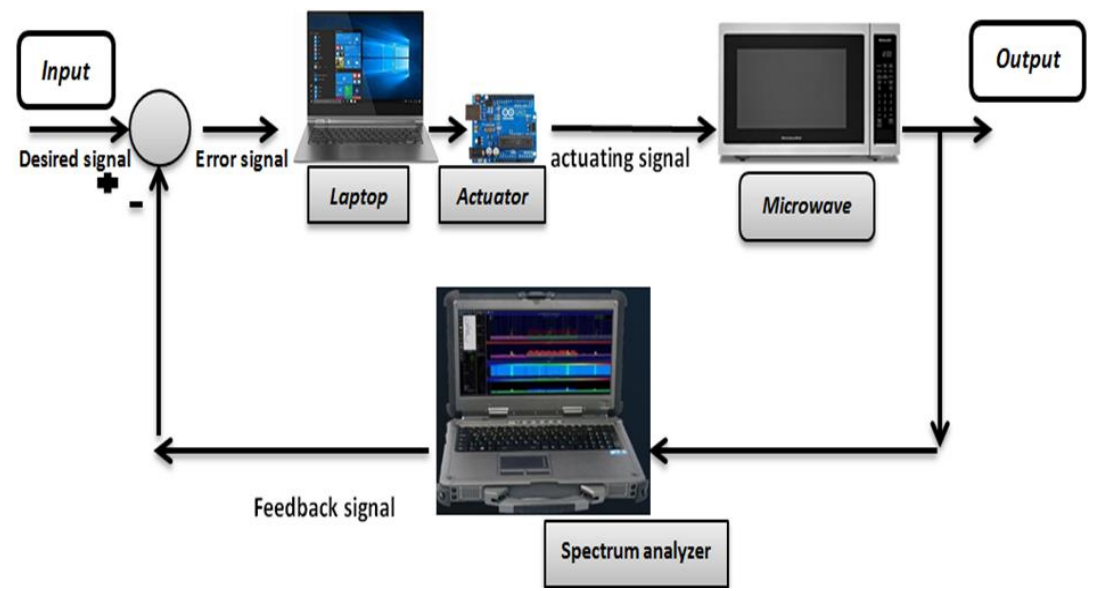

FIG. 2. THE BLOCK DIAGRAM OF AUTOMATIC CONTROL.

The mathematical model that formulates the measured electromagnetic power (microwave leakage power) in relation to incident electromagnetic power is presented in the following:

Power measured by spectrum analyzer, $P_{\text {out }}$ in condition of constant power noise as measured in this research is:

$$
P_{\text {out }}=\left(P_{\text {in }}-P_{\text {abs }}\right) * k * S
$$

The input (source) electromagnetic power, $P_{i n}$ at waveguide incident port is called magnetron power, which is $850 \mathrm{~W}$ in this research and can be represented by average Pointing vector [41].

$\boldsymbol{P}_{\boldsymbol{a b s}}$ is adsorbed power.

$$
P_{\text {in }}=(1 / 2)\left(E_{0}^{2} / Z_{x y}\right)(a . b)
$$

where, $E_{0}$ is electric field intensity, $Z_{x y}$ is transverse wave impedance for TE modes in a rectangular guide [20], $a$ and $b$ are dimensions of wave incident plane in $x$-and $y$-axes [41].

$$
Z_{x y}=E_{0} / H_{0}=Z /\left(1-\left(f_{c} / f\right)^{2}\right)^{1 / 2}
$$

where, $H_{0}$ is magnetic field, $Z$ is intrinsic impedance for medium, $f$ is operating or supplied frequency and $f_{c}$ is cutoff frequency.

$$
\begin{aligned}
& Z=\sqrt{\mu / \varepsilon} \\
& f_{c}=\left(\frac{1}{2 \pi \sqrt{\mu \varepsilon}}\right) \sqrt{(u \pi / a)^{2}+(v \pi / b)^{2}}
\end{aligned}
$$

where, $u$ and $v$ are integer numbers of sinusoidal half wavelength variation in $x$ - and $y$ axes, $\mu$ is a magnetic permeability.

The electromagnetic power that is absorbed by dielectric materials $P_{a b s}$ is dissipated as heat, JHEAT as represented in (9):

where:

$$
P_{a b s}=J H E A T=\left(\frac{1}{2}\right) \omega \varepsilon_{0} \varepsilon_{r}^{\prime}(\tan \delta)|E|^{2} d V
$$

$$
\tan \delta=\left(\varepsilon_{r}^{\prime \prime} / \varepsilon_{r}^{\prime}\right)+\sigma /\left(\omega \varepsilon_{0} \varepsilon_{r}^{\prime}\right)
$$

$k$ : is constant value represents the power attenuation of microwave cavity called attenuator pad. Where, the microwave cavity is a closed surfaces system of a perfect 
electrical conductor works as a Faraday cage [42], which highly attenuates or reduces the microwave leakage to out of microwave oven.

$S$ : is antenna sensitivity or receiver sensitivity which is a receiver's ability to identify and amplify the receiver input signals. It is expressed in $\mathrm{dBm}$. The level of sensitivity of the receiver indicates to the weakest signal a recipient can identify and process. It may be improved by lowering the receiver's noise level and bandwidth [43].

The control process will be done by means of two comparisons to turn off the microwave oven. The first condition: is finding with time till the measured power reaches (- 80) at the frequencies between $(2.46$ - 2.47)(the reason is mentioned in the discussion), with a calculated error rate. When the first condition is met, the program will go to the second condition. The second condition: is finding the higher measured power at the same time as the first condition within frequencies between $2.45-2.46$, as shown in Fig. 3 . When the computer code achieves the two conditions, a signal will be sent from Arduino to a relay to turn off the oven.

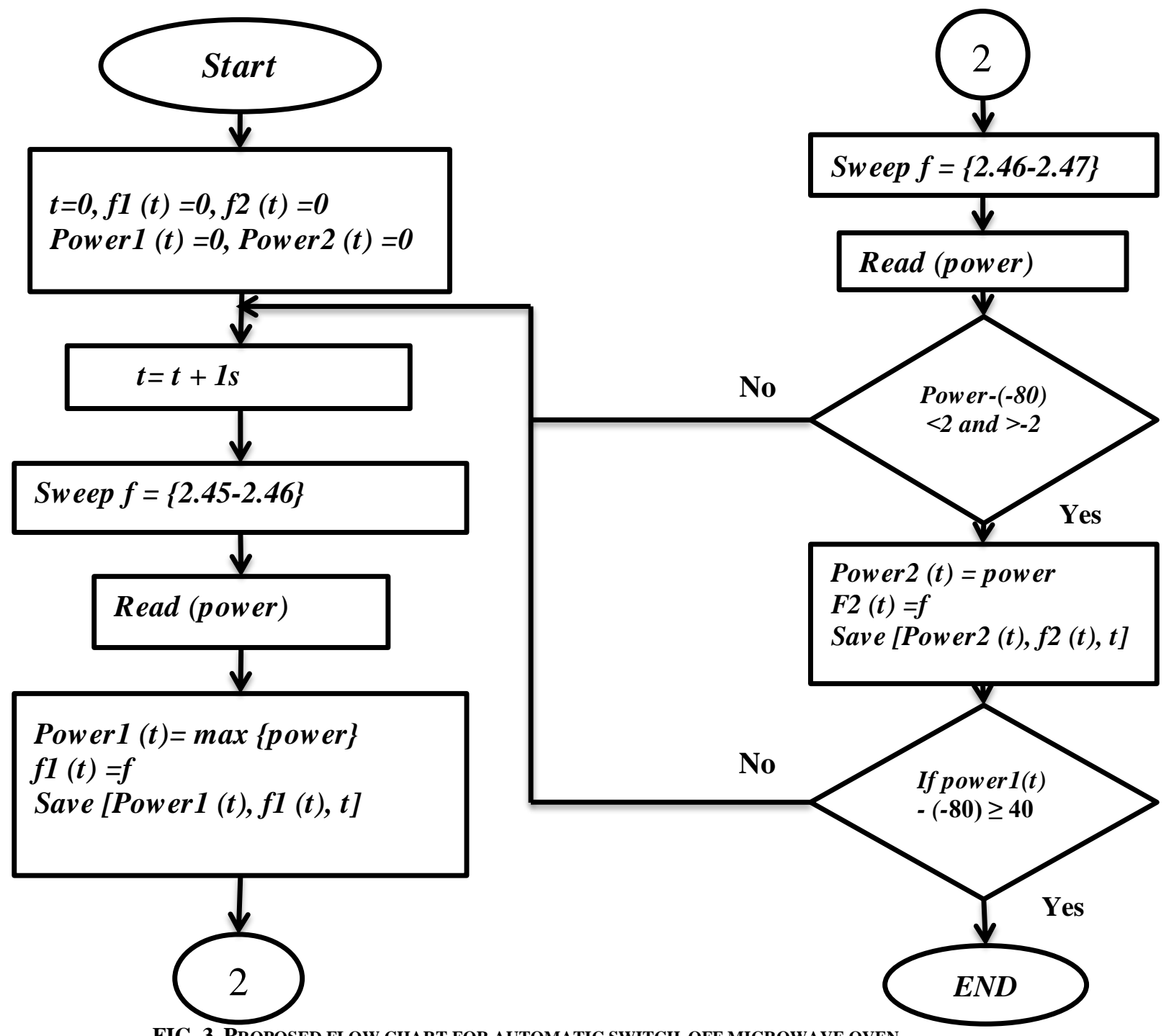

\section{EXPERIMENTAL WORK}

This experiment aims to demonstrate the possibility of measuring the electromagnetic power leakage as an indicator for microwave power inside a microwave oven using an outside antenna linked with a spectrum analyzer. This experiment was not discussed in the 
DOI: https://doi.org/10.33103/uot.ijccce.21.1.4

previous works according to this configuration, which is a part of the concept of the proposed technique. Latterly, further work on the control process depending on the measurement will be done experimentally.

The setup of experimental work is to link the spectrum analyzer (Aaronia, German) with microwave oven (swan, $850 \mathrm{~W}$ microwave power) through antenna (Sunhans, $2.45 \mathrm{GHz}, 6 \mathrm{~dB}$ ) remotely as shown in Fig. 4. Microwave oven was put in isolated room (RF anechoic chamber) as shown in Fig. 5. Anechoic chamber means a box or room without reflections (echoes) from the walls, ceiling, and floor. The walls are treated with fiberglass sound absorbers to suppress echoes. In the RF world, talk about a shielded room, where radio wave absorbers were used to treat the inner surfaces. Typical absorbers could be carbon loaded foam pyramids, or ferrite tiles. This experiment is to demonstrate the changes in the power of signal leaked from microwave oven in three cases. The first case when the microwave oven is off. The second case when the microwave oven is switched on without material inside. In the third case, a material is added inside the running microwave oven. Thus, this experiment is to prove the power of external signal changes with the state of the material inside the microwave oven. Each of the experiments of $200 \mathrm{ml}$ and $400 \mathrm{ml}$ water was repeated three times.

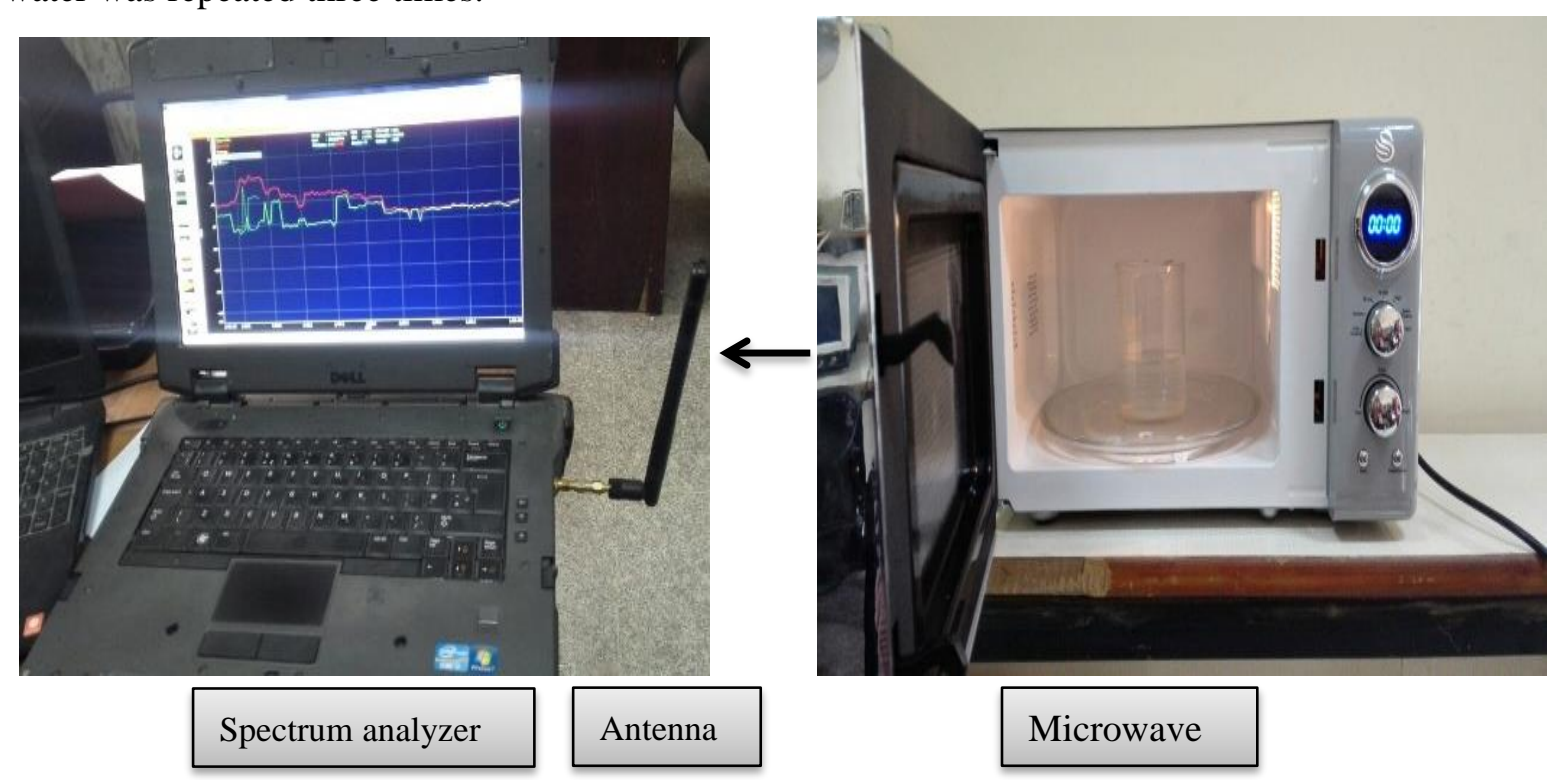

Fig. 4. SPECTRUM ANALYZER CONNECTED WITH MICROWAVE OVEN THROUGH ANTENNA. 
DOI: https://doi.org/10.33103/uot.ijccce.21.1.4

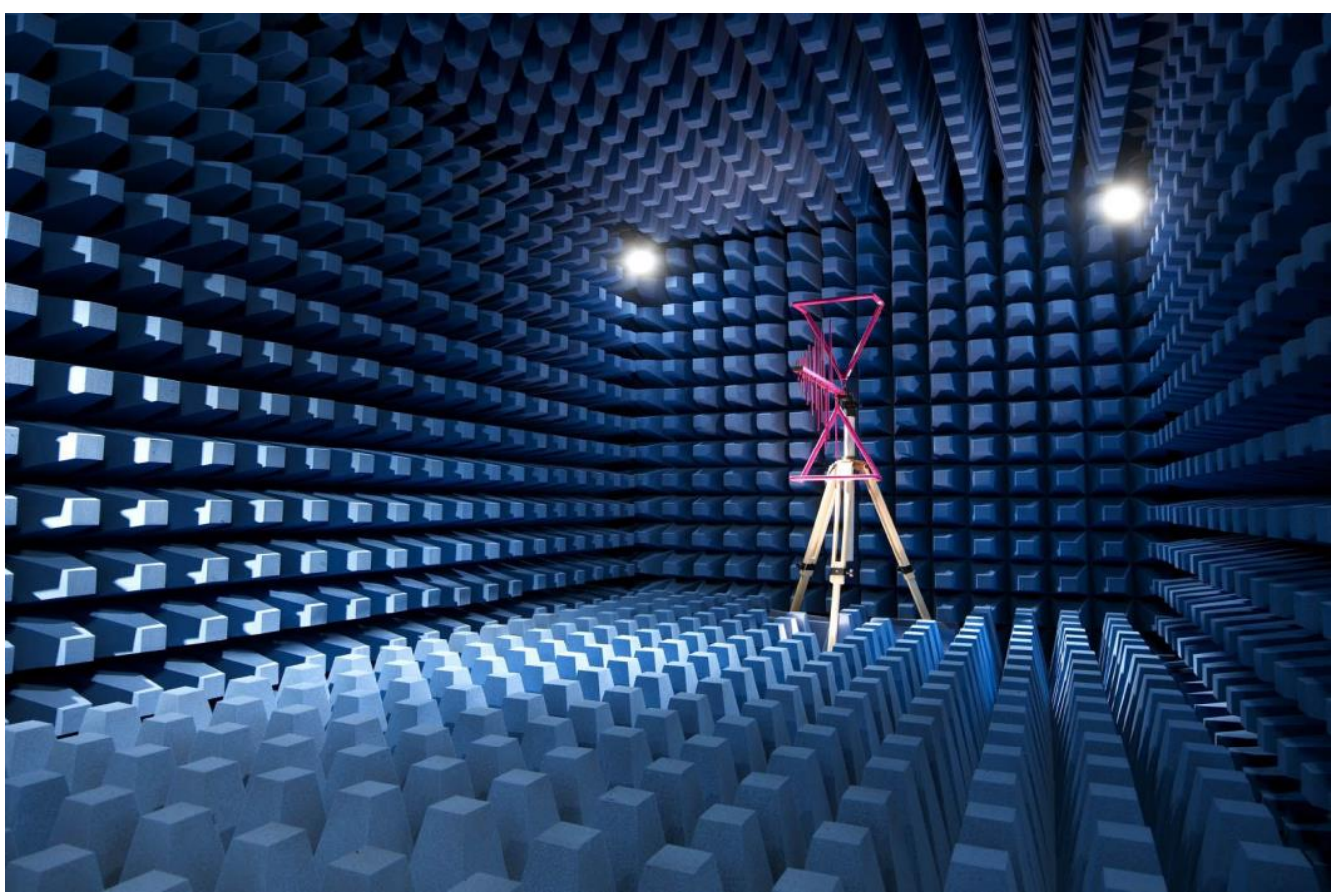

FIG. 5. RF ANECHOIC CHAMBER PREVENTS THE ELECTROMAGNETIC INTERFERENCE BETWEEN TWO OPERATORS [44]

\section{PReliminary ReSUlts}

The preliminary results that are shown in Fig. 6 and Fig. 7, presented the electromagnetic power leakage for frequencies ranged from 2.45 to $2.47 \mathrm{GHz}$ emerged from a magnetron inside a microwave oven that was positioned in RF anechoic chamber.

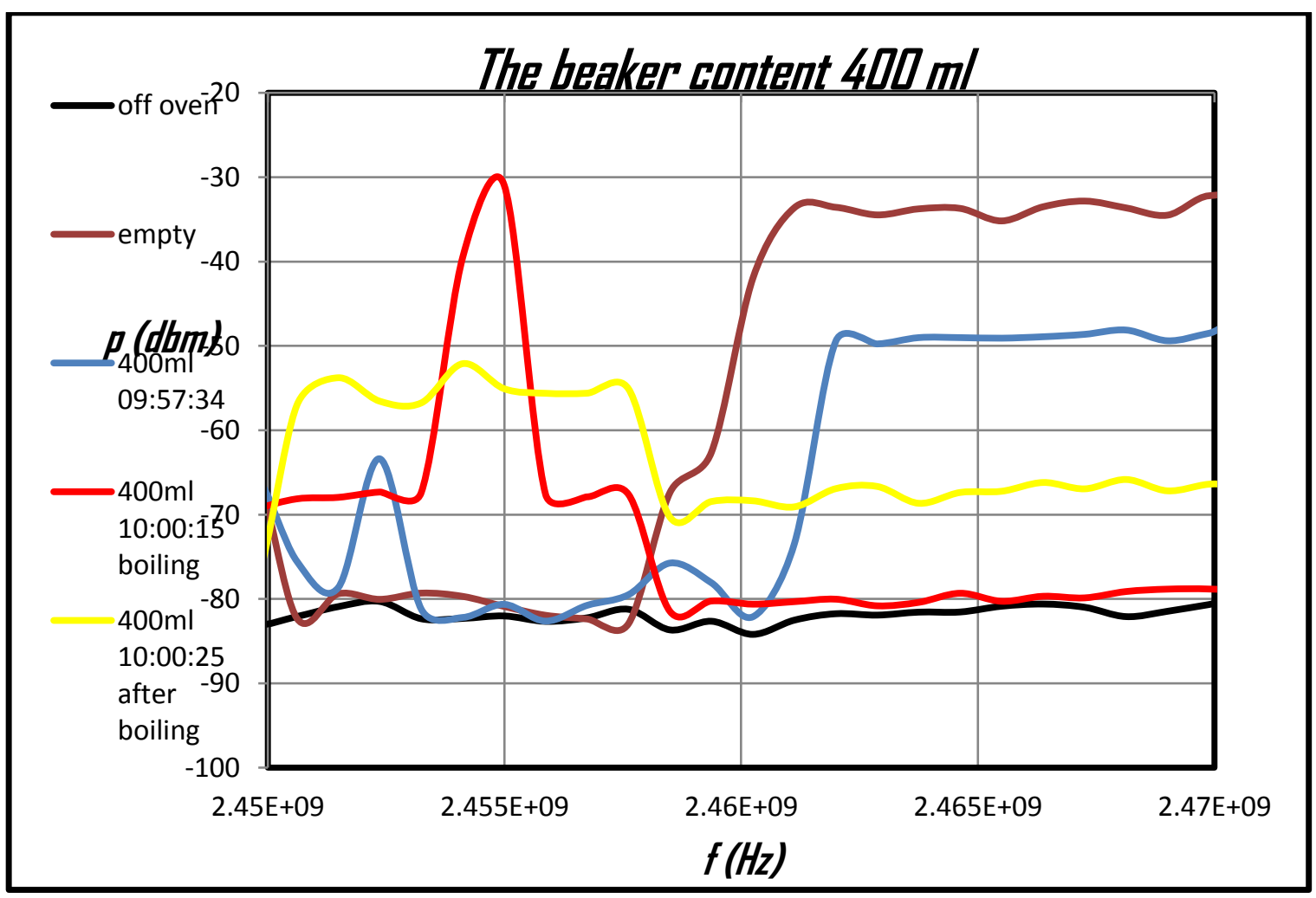

FIG. 6. ELECTROMAGNETIC POWER VS. FREQUENCY MEASURED INSIDE THE MICROWAVE CAVITY FOR 400 ML WATER. 


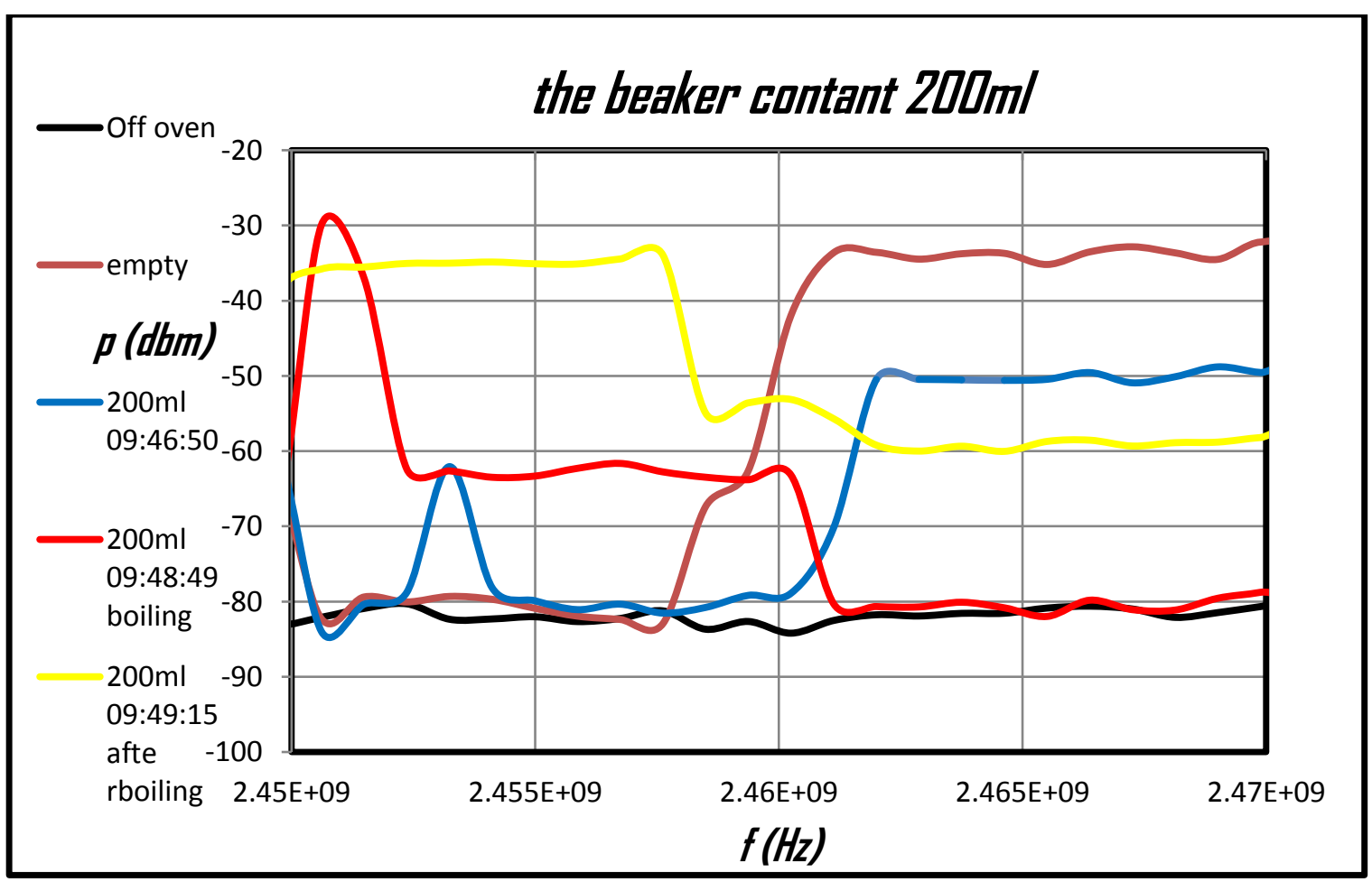

Fig. 7. ELECTROMAGNETIC POWER VS. FREQUENCY MEASURED INSIDE THE MICROWAVE CAVITY FOR 200 ML WATER.

It is noticed from Fig. 6 and Fig. 7 that the black curve, in each of both figures, was fluctuated around $(-80) \mathrm{dBm}$, which represented the power of noise signal during the nonoperation of the microwave oven where the magnetron was switched off.

The brown curve in each of Fig. 6 and Fig. 7 showed the trend when the microwave cavity of the oven was empty while the magnetron was switched on. The value of measured power was between $(-35$ to -25$) \mathrm{dBm}$ for frequencies ranged $(2.46-2.47)$. This value of power represented the amount of power emitted from the magnetron without the existence of a power absorber.

The blue curve in Fig. 6 and Fig. 7 represented the trend when there were $400 \mathrm{ml}$ and $200 \mathrm{ml}$ of water inside the microwave cavity heated for less than one minute. The value of measured power for each of $400 \mathrm{ml}$ and $200 \mathrm{ml}$ water was reduced to about $(-50) \mathrm{dBm}$ for frequencies ranged $(2.46$ - 2.47). It could be noticed that the difference in measured power between the trend of the empty cavity and trend of the presence of $400 \mathrm{ml}$ and $200 \mathrm{ml}$ water during the switched on microwave oven was the value of electromagnetic power absorbed by the dielectric material, which is water. The absorbed electromagnetic energy was dissipated inside the water as thermal energy and increased the water temperature.

The red curve represented the trend of $400 \mathrm{ml}$ and $200 \mathrm{ml}$ water after about 3 and 2 minutes respectively of microwave heating attaining the boiling point. The value of measured power for frequencies ranged $(2.46$ - 2.47) was went back to about $(-80) \mathrm{dBm}$. This phenomenon means all the electromagnetic power was absorbed by water due to the random oscillated motion of water at the boiling state. The difference in boiling time for $200 \mathrm{ml}$ and $400 \mathrm{ml}$ water reverts to the difference in the amount of water.

The yellow curve presented the trend after the boiling, which showed that the measured power of frequencies ranged (2.46 - 2.47) increased to about (-70) $\mathrm{dBm}$ and (-60) $\mathrm{dBm}$ for $400 \mathrm{ml}$ and $200 \mathrm{ml}$ water respectively. The reason for the power increase was attributed to the lack of volume of water due to the water evaporation leading to a lack in the absorbed electromagnetic energy as shown in (9). This resulted to increase in the 
measured power that represented the microwave power leakage as stated in (4). The measurement of leakage power for $400 \mathrm{ml}$ water is less than $200 \mathrm{ml}$ because the absorbed power by the amount of $400 \mathrm{ml}$ water is more than $200 \mathrm{ml}$.

Furthermore, for frequencies $(2.45-2.46)$ that appeared in the brown and blue curves, the value of power was around $(-80) \mathrm{dBm}$, which means no power emission from the magnetron at these frequencies. While, red and yellow curves showed growth in power due to the random oscillated motion of evaporated water that having electrons transferred from an orbit of energy to another, dissipating the thermal energy as electromagnetic energy and this corresponds to what is stated in [45]. The difference in the amount of water leads to some difference in the behavior of measured power as shown in Fig. 6 and Fig. 7. It should be noted that each of the experiments of $200 \mathrm{ml}$ and $400 \mathrm{ml}$ water was repeated three times and the results were identical with an error ratio (4\%).Where the max measured power was between $(-28,-32)$.

\section{CONCLUSIONS}

The current study provided a detailed review of measurement and control on the electromagnetic radiation inside a microwave oven. To automatically control a microwave oven, a new concept is proposed as a control system feedback, which is the measurement of electromagnetic power leakage of the microwave oven. The preliminary results proved that the leakage of electromagnetic power changes with the state/phase of the material inside the microwave oven. These results ensured the possibility of using the proposed technique to solve the problem of microwave power measurement inside the microwave oven.

\section{THE FUTURE WORK}

This work will be continued to connect the microwave oven with a spectrum analyzer and computer via hardware and software interfaces depending on the methodology mentioned in this article. Computer code will be developed to read the measured power and automatically control and switch off the microwave oven.

\section{ACKNOWLEDGEMENT}

The authors Acknowledge Electronic Manufacturing Center, Directorate of Industrial Research and Development, Ministry of Science and Technology, Baghdad, Iraq, for their assistance to conduct this research.

\section{REFERENCES}

[1] M. Heggannavar, P. Mugali, \& S. M. Patil. “ Design And Development Of Magnetron Power Source From Three Phase Supply, ’International Journal of Engineering Research \& Technology (IJERT), Vol.2, No.7, 2013.

[2] T. Santos, M. A.Valente, J. Monteiro, J.Sousa, \& L. C. Costa. "Electromagnetic and thermal history during microwave heating," Applied Thermal Engineering, Vol.31, No.16, pp. 3255-3261, 2011.

[3] H. B. Al-Wakeel, Z. A. Karim, \& H. H. Al-Kayiem "A technique for localized rapid soot oxidation using metal aided microwave radiation, "IEEE Transactions on Microwave Theory and Techniques,Vol. 64, No.1, pp.37-43, 2015.

[4] S. K.Korkua, S.Chandhaket, K. Thinsurat, \& K. Pornbandit, "Design of automatic phase-controlled converter based on temperature for microwave drying system," In 2016 2nd IEEE International Symposium on Robotics and Manufacturing Automation (ROMA) (pp. 1-6). IEEE, September,2016.

[5] Z. Li, G. S. V. Raghavan, \& V. Orsat. "Temperature and power control in microwave drying,"Journal of Food Engineering, Vol.97, No.4, pp.478-483, 2010.

[6] J. P. van der Merwe, \& J. B. De Swardt. " Power control of a domestic microwave oven," In 2017 IEEE AFRICON (pp. 574-578). IEEE, September,2017.

[7] H. B. Al-Wakeel, Z. A. Abdul Karim, \& H. H. Al-Kayiem. "Soot reduction strategy: A review. Journal of Applied Sciences," Vol.12, No. 23, pp. 2338-2345, 2012.

[8] X. Wu. "Experimental and theoretical study of microwave heating of thermal runaway materials," (Doctoral dissertation, Virginia Tech). 2002. 
[9] V. Palma, P. Russo, M. D’Amore, \& P. Ciambelli. “ Microwave regenerated catalytic foam: a more effective way for PM reduction. Topics in catalysis,” Vol.30, No.1-4, pp. 261-264, 2004.

[10] J. Ma, M. Fang, P. Li, B. Zhu, X. Lu, \& N. T. Lau, "Microwave-assisted catalytic combustion of diesel soot," Applied Catalysis A: General, Vol.159, No.1-2, pp.211-228, 1997.

[11] B. G. McConnell, "A coupled heat transfer and electromagnetic model for simulating microwave heating of thin dielectric materials in a resonant cavity," (Doctoral dissertation, Virginia Tech). 1999.

[12] Y. Zhang-Steenwinkel, L. M. Van der Zande, H. L. Castricum, A. Bliek, R. W. Van den Brink, \& G. D. Elzinga, "Microwave-assisted in-situ regeneration of a perovskite coated diesel soot filter, " Chemical engineering science, Vol.60, No.3, pp.797-804, 2005.

[13] D. IghigeanuI, D. Martin, C. OproiuI, E. Manaila, G. Craciun, I. Calinescu, \& E. Zissulescu, "VOC removal by microwave, electron beam and catalyst technique," 2006.

[14] S. M. B. Taslim, \& S. M. R. Tousif, “ Investigation of suitability of operating frequency and electro technical modeling of microwave oven. In 2011 10th International Conference on Environment and Electrical Engineering," (pp. 1-5). IEEE, May,2011.

[15] P. Pesheck, \& M. Lorence, (Eds.). "Development of packaging and products for use in microwave ovens, ” Elsevier. 2009.

[16] Y. J. Woo, S. K. Kim, \& G. H. Cho, "Voltage-clamped class-E inverter with harmonic tuning network for magnetron drive," IEEE Transactions on Circuits and Systems II: express briefs, Vol.53, No. 12, pp.1456-1460, 2006.

[17] J. B. Murphy, J. T. DeBord, E. A. Soller, \& R. W. U.S. Cartwright, "Washington, DC: U.S. Patent and Trademark Office," Patent No.10, pp.584,881, 2020.

[18] K. Nakatani, \& T. Ishizaki, “A 2.4 GHz-band $100 \mathrm{~W}$ GaN-HEMT high-efficiency power amplifier for microwave heating, "Journal of electromagnetic engineering and science, Vol.15, No.2, pp.82-88, 2015.

[19] B. V. Barbadekar, "Microwave controller a new approach in agriculture as pesticide," 2017.

[20] J. D. Kraus and D. A. Fleisch, “Electromagnetics with Applications, 5th ed, ”Singapore: McGraw Hill, 1999.

[21] T. V. C. T. Chan and H. C. Reader, "Understanding microwave heating cavities, " London: Artech House Inc., 2000.

[22] Y. Yuan, S. Liang, Q. Xiong, J. Zhong, \& Z. Wang, "Thermodynamics model based temperature tracking control in microwave heating," Journal of Thermal Science and Technology, Vol.11, No.1, pp.JTST0004-JTST0004, 2016.

[23] H. J. Tyler, \& W. H. Conway, U.S. Patent No. 4,297,557. Washington, DC: U.S. Patent and Trademark Office. 1981.

[24] S. N. A. Alrazaaq, "LabVIEW Based Fuzzy Controller Designed for a Microwave Oven," Tikrit Journal of Engineering Sciences, Vol.23, No.1, pp.61-68, 2016.

[25] S. K. Dubey, N. Narang, P. S. Negi, \& V. N. Ojha, "LabVIEW based Automation Guide for Microwave Measurements, " Springer. 2017.

[26] J. R. Flemish, \& R. L. Haupt, "Optimization of a photonically controlled microwave switch and attenuator,"IEEE transactions on microwave theory and techniques, Vol.58, No.10, pp.2582-2588, 2010.

[27] X. Guo, Z. Gong, Q. Zhong, X. Liang, \& Z. Liu, "A miniaturized reconfigurable broadband attenuator based on RF MEMS switches,” Journal of Micromechanics and Microengineering, Vol.26, No.7, pp.074002, 2016.

[28] W. Adamski, M. Kitlinski, \& W. Rydzkowski, "A set-up to determine energy absorption during microwave heating," In 12th International Conference on Microwaves and Radar. MIKON-98. Conference Proceedings (IEEE Cat. No. 98EX195) (Vol. 2, pp. 633-637). IEEE. May, 1998.

[29] Heba Hadi Ali. "Investigation on the Microwave Power Absorption in Tissues," PhD thesis Mustansiriayah University. Electrical Engineering Department.2019.

[30] D. Abdorahimi, \& A. M Sadeghioon,. "Comparison of radio frequency path loss models in soil for wireless underground sensor networks," Journal of Sensor and Actuator Networks, Vol.8, No.2, pp.35, 2019.

[31] H. B. Al-Wakeel, Z. A. A. Karim, H. H. Al-Kayiem, \& H. Fawad, "Numerical simulation of high frequency electromagnetic wave in microwave cavity for soot oxidation," Applied Mechanics and Materials (Vol. 459, pp. 310-318). Trans Tech Publications Ltd. 2014.

[32] G. D. Rosa, \& N. B. Carvalho, "Microwave oven field detector probe," In 2018 IEEE Wireless Power Transfer Conference (WPTC) (pp. 1-4). IEEE.) June ,,$\cdot 1 \wedge$.

[33] D. E. Panait, A. C. Jufa, L. Floroian, A. M. Pascu, M. Badea, M. Popa,... \& S. Bungau, “ Electromagnetic Pollution of the Environment Due Leakage Radiation from Microwave Ovens, ” 2019.

[34] P. Lopez-Iturri, S. de Miguel-Bilbao, E. Aguirre, L. Azpilicueta, F. Falcone, \& V. Ramos, (2015). “Estimation of radiofrequency power leakage from microwave ovens for dosimetric assessment at nonionizing radiation exposure levels, " BioMed research international, 2015. 
[35] A. J. Salim, J. K. Ali, \& H. S. Ahmed, "Design of fractal-based bandstop filter for microwave radiation leakage reduction," Engineering and Technology Journal, Vol.35, No.1 Part (A) Engineering, pp.16-23, 2017.

[36] M. B. N. Shah, N. Zailany, A. F. Z. Abidin, M. F. Halim, K. A. Annuar, A. H. Azahar, \& M. F. Yaakub, "PIDbased temperature control device for electric kettle,"International Journal of Electrical and Computer Engineering, Vol.9, No.3, pp.1683-1693, 2019.

[37] L. Huang, \& J. Sites, "Automatic control of a microwave heating process for in-package pasteurization of beef frankfurters," Journal of food engineering, Vol.80, No.1, pp.226-233, 2007.

[38] Y. Yuan, S. Liang, S. Gan, J. Zhong, Z. Wang, \& Q. Xiong, "Power density and sample temperature control for microwave heating system, "In Proceeding of the 11th World Congress on Intelligent Control and Automation (pp. 6151-6156). IEEE . June, Y. I.

[39] W. M. Cheng, "Microwave Power Control Strategies on the Drying Process," (Doctoral dissertation, McGill University). 2004.

[40] Z. Li, "Design of a Microcontroller-based, Power Control System for Microwave Drying," (Doctoral dissertation, McGill University). 2004.

[41] J. Jin, "The finite element method in electromagnetics," 3rd ed. New York: Wiley, 2014.

[42] H. B. Al-Wakeel, Z. A. Karim, \& H. H. Al-Kayiem, “ Optimizing electro-thermo Helds for soot oxidation using microwave heating and metal,” In IOP Conference Series: Materials Science and Engineering (Vol. 78, No. 1, p. 012018). IOP Publishing. 2015.

[43] Q. Gu, "RF system design of transceivers for wireless communications," Springer Science \& Business Media. 2006.

[44] F. O. Ehiagwina, A. S. Afolabi, N. T. Surajudeen-Bakinde, \& O. A. Fakolujo, "Sensitivity degradation and antenna isolation analyses for a multi-operator global systems for mobile communication base transceiver stations, ” Nigerian Journal of Technology, Vol.38, No.1, pp.177-184, 2019.

[45] J. D. Olsen, \& K. T. McDonald,"Classical lifetime of a bohr atom," Joseph Henry Laboratories, Princeton University,2005. 